\title{
Laparoscopic Anderson-Hynes procedure as a treatment of ureteropelvic junction obstruction caused by fibroepithelial polyp
}

\author{
Tomasz Szydelko ${ }^{1,2}$, Tomasz Tuchendler ${ }^{3}$, Adam Litarski ${ }^{3}$, Grzegorz Urbańczyk ${ }^{3}$, Wojciech Apoznański, Dariusz Janczak ${ }^{5}$ \\ ${ }^{1}$ Clinical Department of Urology, $4^{\text {th }}$ Clinical Military Hospital, Wroclaw, Poland \\ 2Department of Palliative Care Nursing, Wroclaw Medical University, Wroclaw, Poland \\ ${ }^{3}$ Department of Urology, $4^{\text {th }}$ Clinical Military Hospital, Wroclaw, Poland \\ ${ }^{4}$ Department of Pediatric Surgery and Urology, Wroclaw Medical University, Wroclaw, Poland \\ ${ }^{5}$ Department of Clinical Procedures, Wroclaw Medical University, Wroclaw, Poland
}

Videosurgery Miniinv 2013; 8 (4): 361-363 DOI: 10.5114/wiitm.2011.35636

\begin{abstract}
Fibroepithelial polyps (FEPS) are rare benign ureteral neoplasms of mesodermal origin. As an intrinsic cause of ureteropelvic junction obstruction (UPJO) they are very uncommon. Excretory urography and diuretic renography used by many urologists to diagnose a UPJO may not detect the ureteral polyp. We present a case of ureteropelvic junction obstruction caused by a fibroepithelial polyp, which was not diagnosed preoperatively. The patient underwent successful laparoscopic excision of the polyp with Anderson-Hynes pyeloplasty. We believe that laparoscopy is a useful, minimally invasive operative technique in the treatment of FEPs and may be considered a procedure of choice in large polyps, in which cases the endoscopic procedure is difficult or impossible to perform.
\end{abstract}

Key words: ureteropelvic junction, fibroepithelial polyp, laparoscopy.

\section{Introduction}

Fibroepithelial polyps (FEPs) are rare benign ureteral neoplasms of mesodermal origin. They are usually present in the third and fourth decades of age, but they may occur in any age - from the newborn to the elderly $[1,2]$. The FEPs are diagnosed more frequently in males than in females, in the left ureter twice as often as in the right [3]. They usually occur in the proximal third of the ureter, although they can form in any place of the urinary tract [3].

The FEPs located in the ureteropelvic junction are very rarely the cause of obstruction. Excretory urography and diuretic renography used by many urologists to diagnose a ureteropelvic junction obstruction (UPJO) may not detect the ureteral polyp.
We present a case of UPJO caused by a fibroepithelial polyp, which was not diagnosed preoperatively. The patient underwent successful laparoscopic excision of the polyp with Anderson-Hynes pyeloplasty.

\section{Case report}

A 31-year-old woman was admitted to our institution with the diagnosis of left UPJO. She had suffered from recurrent left flank pain for 10 months. Ultrasonography revealed left-sided hydronephrosis. A renogram curve showed a plateau-like third phase, with no reaction following furosemide administration. A split renal function for the left kidney was $49.3 \%$. Excretory urography revealed left 


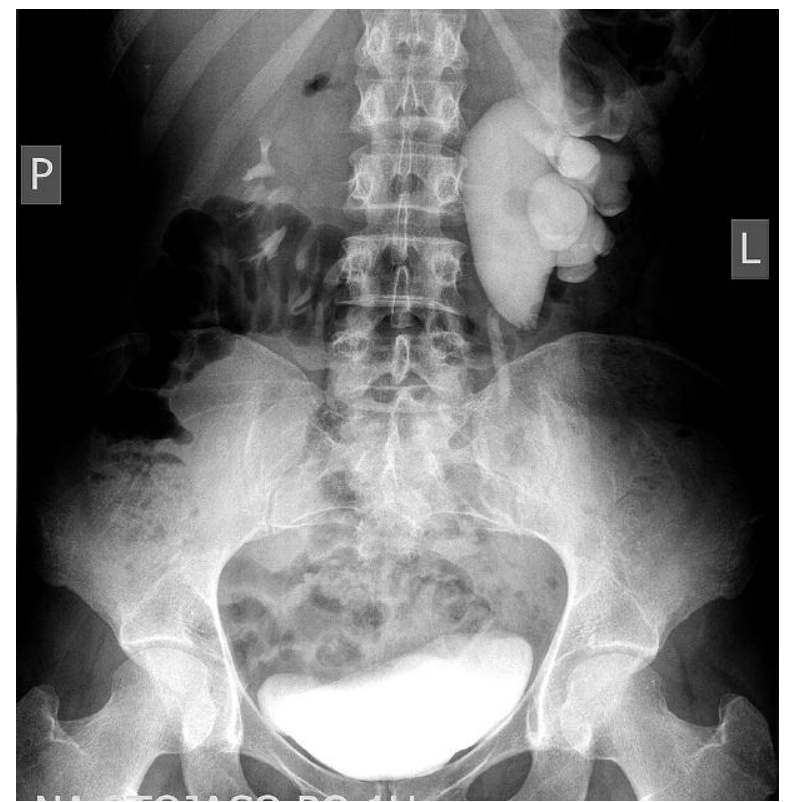

Photo 1. Excretory urography showing left ureteropelvic junction obstruction caused by a high insertion of the ureter and/or crossing vessel

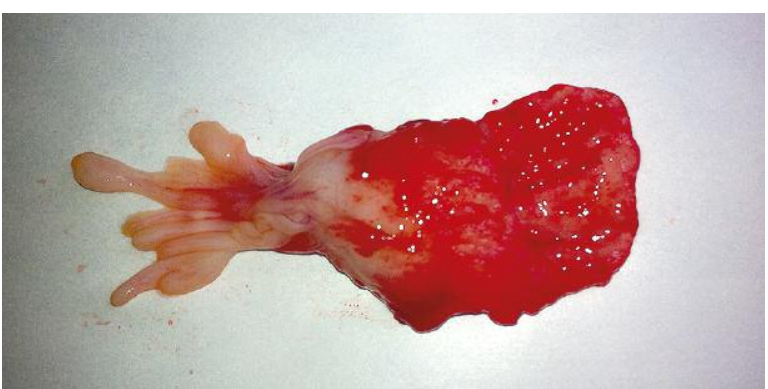

Photo 2. Ureteropelvic junction with a fibroepithelial polyp

hydronephrosis caused probably by a high insertion of the ureter and/or crossing vessel (Photo 1). The decision was made to perform laparoscopic pyeloplasty.

The procedure was started with a 6-Fr double-J stent placement. The patient was placed in a $45^{\circ}$ flank position. A Hasson technique was used to insert the first $10 \mathrm{~mm}$ trocar below the umbilicus. Two $5 \mathrm{~mm}$ trocars were inserted below the costal margin and a $10 \mathrm{~mm}$ trocar was placed laterally to the umbilicus. The renal pelvis and the proximal ureter were mobilized. There was no crossing vessel around the ureteropelvic junction (UPJ). The renal pelvis was incised and a polypoid mass was found in the ureteropelvic junction. Part of the redundant

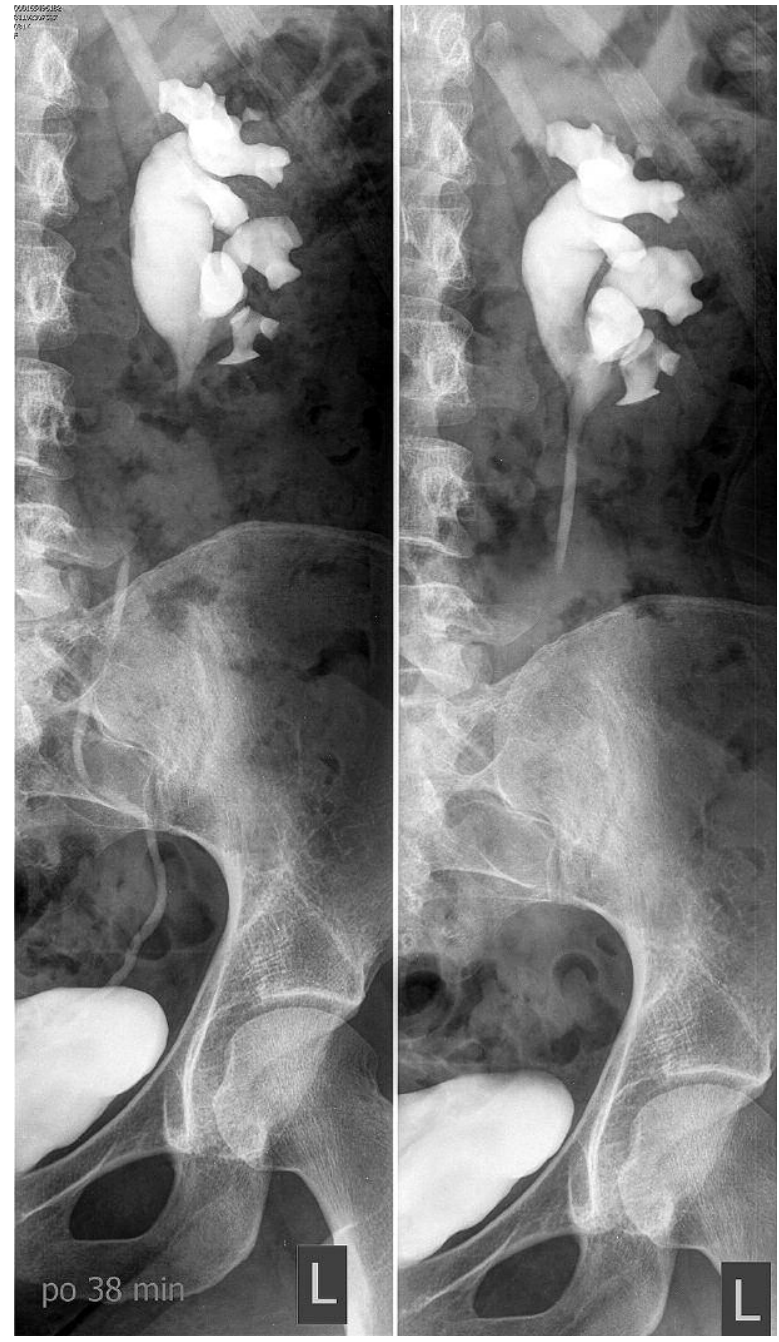

Photo 3. Excretory urography showing a patent ureteropelvic junction - 10 months after the operation

renal pelvis was excised and the ureter was spatulated. The pedicle of the polyp was identified and the ureter was cut off about $1 \mathrm{~cm}$ below (Photo 2). The Anderson-Hynes pyeloplasty was completed using two running sutures (Vicryl 4-0, Johnson \& Johnson Intl, St-Stevens-Woluwe, Belgium). The suction drain was left in place and a Foley catheter was left in the bladder. There were no postoperative complications. A double-J stent was removed 4 weeks after the procedure. Ten months after the operation diuretic renography revealed no obstructive pattern and an excretory urography showed a patent UPJ (Photo 3). Pathologic findings of the resected mass demonstrated a fibroepithelial polyp. The surgical margins were negative - the excision was complete. 


\section{Discussion}

The UPJO may be caused by intrinsic or extrinsic factors. A fibroepithelial polyp as an intrinsic factor is found extremely rarely. In a study of Adey et al. FEPs were found in nine cases of 1710 pyeloplasties performed for UPJO in children [4]. More commonly fibroepithelial polyps are found in young adults [1]. The patients usually suffer from intermittent flank pain or haematuria. Preoperative radiographic diagnosis of FEPs based on excretory urography could be challenging, because they usually present as a filling defect, which may be caused by blood clots, radiolucent calculi, neoplasm or a crossing vessel [5]. To determine the polyps multidetector computed tomography (CT) scanning or virtual CT ureteroscopy can be helpful but neither of these techniques is routinely used in patients with suspected UPJO [6, 7]. In our case the results of excretory urography (IVU) and diuretic renography were typical of UPJO; hence we did not perform any other diagnostic procedures. Even though ureteroscopic endopyelotomy has been widely reported as effective, we believe that laparoscopic pyeloplasty is the most effective procedure in the treatment of ureteropelvic junction obstruction, so a decision was made to operate on the patient laparoscopically [8]. Preoperative diagnosis of a fibroepithelial polyp may result in the decision to undertake the ureteroscopic treatment $[9,10]$. Yet, there are reports in the literature that indicate that endoscopic procedures may not accomplish a total resection of the lesion and are not effective enough to resect the base of the polyp [11]. In our case the laparoscopic procedure made it possible to observe the root of the polyp, which allowed a complete resection with negative surgical margins. We believe that laparoscopy is a useful, minimally invasive operative technique in the treatment of FEPs and may be considered a procedure of choice in large polyps, in which cases the endoscopic procedure is difficult or impossible to perform.

In conclusion, FEPs should be taken into account as a possible cause of UPJO, especially in young adults. Resection of the polyp in the UPJ with Anderson-Hynes plasty is a safe and effective procedure and may be performed laparoscopically.

\section{References}

1. Williams TR, Wagner BJ, Corse WR, et al. Fibroepithelialpolyps of the urinarytract. Abdom Imaging 2002; 27: 217-21.
2. Childs MA, Umbreit EC, Krambeck AE, et al. Fibroepithelial polyps of the ureter: a single-institutional experience. J Endourol 2009; 23: $1415-9$

3. Bhalla RS, Schulsinger DA, Wasnick RJ. Treatment of bilateral fibroepithelial polyps in a child. J Endourol 2002; 16: 581-2.

4. Adey GS, Vargas SO, Retik AB, et al. Fibroepithelial polyps causing ureteropelvic junction obstruction in children. J Urol 2003; 169: 1834-6.

5. Iwatsuki S, Kojima Y, Mizuno K, et al. Laparoscopic management for fibroepithelial polyp causing ureteropelvic junction obstruction in a child. Urology 2010; 76: 146-8.

6. Sauer B, Flocquet M, Batch T, et al. Vascular renal anatomy and the ureteropelvic junction: preoperative multidetector CT scanning with split-bolus injection as a predictor of laparoscopic findings. J Endourol 2008; 22: 13-8.

7. Chou CP, Wu TT, Levenson RB, et al. Ureteral fibroepithelial polyp diagnosed preoperatively on virtual CT ureteroscopy. Abdom Imaging 2007; 32: 421-3.

8. Wu Z, Feng C, Ding Q, et al. Ureteroscopic holmium:YAG laser endopyelotomy is effective in distinctive ureteropelvic junction obstructions. Videosurgery Miniinv 2011; 6: 144-9.

9. Carey RI, Bird VG. Endoscopic management of 10 separate fibroepithelial polyps arising in a single ureter. Urology 2006; 67: 413-5.

10. Lam JS, Bingham JB, Gupta M. Endoscopic treatment of fibroepithelial polyps of the renal pelvis and ureter. Urology 2003; 62: 810-3.

11. Romesburg JW, Stein RJ, Desai MM, et al. Treatment of child with bilateral ureteropelvic junction obstruction due to fibroepithelial polyps and review of the literature. Urology 2009; 73: e9929.11.

Received: 21.02.2013, accepted: 5.05.2013. 